\title{
CORRESPONDENCE
}

\section{RE: Primary Tumor Location as a Prognostic Factor in Metastatic Colorectal Cancer}

\author{
Felice N. van Erning, Marloes A. Elferink, Amanda C. R. K. Bos, Valery E. P. P. Lemmens \\ Affiliations of authors: Department of Research, Netherlands Comprehensive Cancer Organisation (IKNL), Utrecht, the Netherlands (FNvE, MAE, ACRKB, VEPPL); \\ Department of Public Health, Erasmus MC University Medical Centre, Rotterdam, the Netherlands (FNvE, VEPPL). \\ Correspondence to: Felice N. van Erning, MSc, Netherlands Comprehensive Cancer Organisation (IKNL), PO Box 231, 5600 AE Eindhoven, the Netherlands (e-mail: \\ f.vanerning@iknl.nl)
}

With interest we read the paper by Loupakis et al. (1), in which the prognostic impact of primary tumor location in metastatic colorectal cancer (CRC) was investigated. They found that patients with left-sided tumors had a lower risk of death and progression as compared with patients with right-sided tumors. We repeated the analyses for metastatic CRC patients and additionally investigated whether survival outcomes are also more favorable for patients with left-sided tumors as compared with right-sided tumors in case of nonmetastatic CRC.

Data were retrieved from the Netherlands Cancer Registry. All CRC patients diagnosed in the Netherlands between 2003 and 2008 with stage I to IV and who underwent resection were included. In line with Loupakis et al. (1), we classified tumors proximal to the splenic flexure as right sided and tumors distal to the splenic flexure as left sided. Crude five-year overall survival (OS) was calculated using Kaplan-Meier curves. OS was defined as the time from diagnosis to death or last follow-up date. Multivariable Cox regression was used to discriminate independent risk factors for death. Additionally, we analyzed recurrence-free survival (RFS) for a subgroup of nonmetastatic CRC patients diagnosed in the southern Netherlands for whom data on diagnosis of distant recurrences were retrospectively collected in 2010 and 2011. RFS was defined as the time from diagnosis to recurrence or death, whichever occurred first, or last follow-up date for patients without recurrence or death.

We identified 7104 patients with metastatic CRC, of whom 4158 (58.5\%) had a left-sided tumor. In line with Loupakis et al. (1), we found that patients with left-sided tumors had favorable OS as compared with patients with right-sided tumors (crude 5 -year Os $16 \%$ vs $10 \%$, adjusted hazard ratio $[\mathrm{HR}]=0.80,95 \%$ confidence interval $[\mathrm{CI}]=0.76$ to $0.84, \mathrm{P}<.0001$ ).

We also included 43482 patients with nonmetastatic CRC, of whom 28131 (64.7\%) had a left-sided tumor. The subgroup consisted of 5457 patients, of whom 3572 (65.5\%) had a left-sided tumor. As shown in Table 1, demographic and clinical characteristics differed by tumor location. Table 2 shows the survival outcomes. For the total nonmetastatic CRC population, crude fiveyear OS was higher for patients with left-sided tumors than for patients with right-sided tumors. However, in multivariable analysis, patients with left-sided tumors had a similar risk of death as compared with patients with right-sided tumors. In the subgroup of patients with data on distant recurrences, crude five-year OS was again higher for patients with left-sided tumors than for patients with right-sided tumors, but crude five-year RFS did not differ between patients with left-sided and right-sided tumors. In multivariable analyses, a trend was observed towards inferior OS for patients with left-sided tumors as compared with patients with right-sided tumors. RFS was inferior for patients with leftsided tumors as compared with patients with right-sided tumors.

Contrary to the findings for patients with metastatic CRC, we found on a population-based level that for patients with nonmetastatic CRC left-sided tumor location showed similar OS and unfavorable RFS as compared with right-sided tumor location, even after adjustment for differences in demographic and clinical characteristics between both tumor locations.

\section{Funding}

This work was supported by the Netherlands Organisation for Health Research and Development (ZonMw) (grant numbers 113102004, 152002012).

\section{Reference}

1. Loupakis F, Yang D, Yau L, et al. Primary tumor location as a prognostic factor in metastatic colorectal cancer. J Natl Cancer Inst. 2015;107(3):dju427 doi:10.1093/jnci/dju427 
Table 1. Demographic and clinical characteristics of M0 patients by primary tumor location*

\begin{tabular}{|c|c|c|c|c|}
\hline \multirow[b]{2}{*}{ Characteristic } & \multicolumn{2}{|c|}{ National level } & \multicolumn{2}{|c|}{$\begin{array}{l}\text { Regional level with data } \\
\text { on distant recurrences }\end{array}$} \\
\hline & $\begin{array}{l}\text { Patients with } \\
\text { right-sided tumors } \\
(\mathrm{n}=15351) \\
\text { No. }(\%)\end{array}$ & $\begin{array}{l}\text { Patients with } \\
\text { left-sided tumors } \\
\text { (n=28 131) } \\
\text { No. (\%) }\end{array}$ & $\begin{array}{l}\text { Patients with } \\
\text { right-sided tumors } \\
(\mathrm{n}=1885) \\
\text { No. }(\%)\end{array}$ & $\begin{array}{l}\text { Patients with } \\
\text { left-sided tumors } \\
(\mathrm{n}=3572) \\
\text { No. }(\%)\end{array}$ \\
\hline \multicolumn{5}{|l|}{ Sex } \\
\hline Male & $6795(44.3)$ & $16230(57.7)$ & $841(44.6)$ & $2063(57.8)$ \\
\hline Female & $8556(55.7)$ & $11901(42.3)$ & $1044(55.4)$ & $1509(42.2)$ \\
\hline$P$ & $<.0001$ & & $<.0001$ & \\
\hline \multicolumn{5}{|l|}{ Age, y } \\
\hline$\leq 50$ & $672(4.4)$ & $1899(6.8)$ & $87(4.6)$ & $233(6.5)$ \\
\hline $51-65$ & $3218(21.0)$ & $9146(32.5)$ & 413 (21.9) & $1206(33.8)$ \\
\hline$>65$ & $11461(74.6)$ & $17086(60.7)$ & $1385(73.5)$ & $2133(59.7)$ \\
\hline$P$ & $<.0001$ & & $<.0001$ & \\
\hline \multicolumn{5}{|l|}{ Comorbidity } \\
\hline None & not available & not available & $513(27.2)$ & $1203(33.7)$ \\
\hline 1 & & & $523(27.8)$ & $1044(29.2)$ \\
\hline$\geq 2$ & & & $733(38.9)$ & $1115(31.2)$ \\
\hline Unknown & & & $116(6.1)$ & $210(5.9)$ \\
\hline$P$ & & & $<.0001$ & \\
\hline \multicolumn{5}{|l|}{ Stage at diagnosis } \\
\hline I & $2334(15.2)$ & $8164(29.0)$ & $275(14.6)$ & $1074(30.1)$ \\
\hline II & 7471 (48.7) & 10097 (35.9) & $938(49.8)$ & $1313(36.7)$ \\
\hline III & $5546(36.1)$ & $9870(35.1)$ & $672(35.6)$ & $1185(33.2)$ \\
\hline$P$ & $<.0001$ & & $<.0001$ & \\
\hline \multicolumn{5}{|l|}{ Mucinous histology } \\
\hline Yes & 3356 (21.9) & $2566(9.1)$ & $352(18.7)$ & $286(8.0)$ \\
\hline No & 11995 (78.1) & 25565 (90.9) & $1533(81.3)$ & 3286 (92.0) \\
\hline$P$ & $<.0001$ & & $<.0001$ & \\
\hline \multicolumn{5}{|l|}{ Differentiation grade } \\
\hline Well/moderate & $10538(68.6)$ & $19770(70.3)$ & $1292(68.6)$ & $2534(70.9)$ \\
\hline Poor/undifferentiated & $3435(22.4)$ & $2774(9.9)$ & $451(23.9)$ & $336(9.4)$ \\
\hline Unknown & $1378(9.0)$ & 5587 (19.8) & $142(7.5)$ & $702(19.7)$ \\
\hline$P$ & $<.0001$ & & $<.0001$ & \\
\hline \multicolumn{5}{|l|}{ Adjuvant chemotherapy } \\
\hline Yes & $3345(21.8)$ & $5075(18.0)$ & $403(21.4)$ & $570(16.0)$ \\
\hline No & $12006(78.2)$ & $23056(82.0)$ & $1482(78.6)$ & $3002(84.0)$ \\
\hline$P$ & $<.0001$ & & $<.0001$ & \\
\hline \multicolumn{5}{|l|}{ Neo-adjuvant radiotherapy } \\
\hline Yes & $10(0.1)$ & $8226(29.2)$ & $3(0.2)$ & $1184(33.2)$ \\
\hline No & 15341 (99.9) & $19905(70.8)$ & $1882(99.8)$ & $2388(66.8)$ \\
\hline$P$ & $<.0001$ & & $<.0001$ & \\
\hline \multicolumn{5}{|c|}{ Neo-adjuvant chemoradiation } \\
\hline Yes & $6(0.1)$ & $1933(6.9)$ & $3(0.2)$ & $282(7.9)$ \\
\hline No & 15345 (99.9) & $26198(93.1)$ & $1882(99.8)$ & $3290(92.1)$ \\
\hline$P$ & $<.0001$ & & $<.0001$ & \\
\hline \multicolumn{5}{|l|}{ Period of diagnosis } \\
\hline 2003-2005 & 7255 (47.3) & $13426(47.7)$ & $862(45.7)$ & 1738 (48.7) \\
\hline $2006-2008$ & $8096(52.7)$ & $14705(52.3)$ & $1023(54.3)$ & $1834(51.3)$ \\
\hline$P$ & .3525 & & .0395 & \\
\hline
\end{tabular}

${ }^{*} \mathrm{P}$ values were based on $\mathrm{X}^{2}$ tests. 
Table 2. Overall and recurrence-free survival outcomes of M0 patients by primary tumor location

\begin{tabular}{|c|c|c|c|c|c|c|}
\hline \multirow[b]{2}{*}{ Demographic level } & \multicolumn{3}{|c|}{ Overall survival } & \multicolumn{3}{|c|}{ Recurrence-free survival } \\
\hline & $\begin{array}{l}\text { Crude 5-y } \\
\text { percentage }\end{array}$ & $\begin{array}{c}\text { Hazard } \\
\text { ratio }(95 \% \mathrm{CI})\end{array}$ & $P$ & $\begin{array}{l}\text { Crude 5-y } \\
\text { percentage }\end{array}$ & $\begin{array}{c}\text { Hazard } \\
\text { ratio }(95 \% \mathrm{CI})\end{array}$ & $P$ \\
\hline \multicolumn{7}{|l|}{ National level* } \\
\hline \multicolumn{7}{|l|}{ Tumor location } \\
\hline Right-sided & 61 & 1.00 (reference) & .4179 & N/A & N/A & N/A \\
\hline Left-sided & 68 & 0.99 (0.95 to 1.02$)$ & & & & \\
\hline \multicolumn{7}{|l|}{ Regional level $\dagger$} \\
\hline \multicolumn{7}{|l|}{ Tumor location } \\
\hline Right-sided & 64 & 1.00 (reference) & .0508 & 61 & 1.00 (reference) & .0111 \\
\hline Left-sided & 68 & $1.11(1.00$ to 1.23$)$ & & 61 & $1.14(1.03$ to 1.27$)$ & \\
\hline
\end{tabular}

* Hazard ratio adjusted for sex, age, stage at diagnosis, mucinous histology, differentiation grade, adjuvant chemotherapy, neo-adjuvant radiotherapy, neo-adjuvant chemoradiation, and period of diagnosis. $P$ value indicates significance of the hazard ratios. $\mathrm{CI}=$ confidence interval; N/A = not available.

† Hazard ratios adjusted for all variables listed under * and additionally for comorbidity. 